\title{
Application of Sliding-Mode Control to the Design of a Buck-Based Sinusoidal Generator
}

\author{
Domingo Biel, Member, IEEE, Enric Fossas, Francisco Guinjoan, Member, IEEE, Eduard Alarcón, Member, IEEE, \\ and Alberto Poveda, Member, IEEE
}

\begin{abstract}
This paper is devoted to the design of a sliding-mode control scheme for a buck-based inverter, with programmable amplitude, frequency, and dc offset, with no external sinusoidal reference required. A general procedure for obtaining an autonomous (time independent) switching surface from a time-dependent one is presented. For this surface, the system exhibits a zeroth-order dynamics in sliding motion. On the other hand, from the sliding-domain analysis, a set of design restrictions is established in terms of the inverter output filter Bode diagram and the output signal parameters (amplitude, frequency and dc offset), facilitating the subsequent design procedure. The control scheme is robust with respect to both power-stage parameter variations and external disturbances and can be implemented by means of conventional electronic circuitry. Simulations and experimental results for both reactive and nonlinear loads are presented.
\end{abstract}

Index Terms-Sinusoidal generation, sliding-mode control, switching converters.

\section{INTRODUCTION}

$\mathbf{P}$ OWER conditioning systems are often designed to supply an ac load from a dc source. The uninterruptible power supplies (UPSs), photovoltaic systems (PVs) connected to the utility grid, or ac power sources constitute the most classical applications. The design of such systems has to achieve a behavior as close as possible to ideal voltage or current sources, in the sense of fast transient response to load variations and steady-state accuracy. The UPS and grid-connected PV systems have to be controlled in such a way that the output voltage or the output current should be in phase with an external sinusoidal reference proportional to the utility grid. For this reason, this external sinusoidal reference is included in the control loop. In these cases, the control loop design is based on the minimization of the error defined as the difference between the output signal and the reference one. This control policy is known as a "tracking" control scheme, in the sense that the output signal is

Manuscript received February 27, 2000; revised December 16, 2000. Abstract published on the Internet February 15, 2001. This work was supported in part by the Comisión Interministerial de Ciencia y Tecnología (CICYT) under Project TAP97-0969-CO3-03/01. This paper was presented in part at IEEE ISIE'99, Bled, Slovenia, July 12-16, 1999.

D. Biel is with the Departamento de Ingenieria Electrónica at the Escuela Universitaria Politècnica de Vilanova i la Geltrú, Universitat Politècnica de Catalunya, 08800 Vilanova i la Geltrú, Spain (e-mail: biel@eel.upc.es).

E. Fossas is with the Institut d'Organització i Control de Sistemes Industrials, Universitat Politècnica de Catalunya, 08028 Barcelona, Spain.

F. Guinjoan, E. Alarcón, and A. Poveda are with the Departamento de Ingenieria Electrónica, Escuela Técnica Superior de Ingenieros de Telecomunicación de Barcelona, Universitat Politècnica de Catalunya, 08034 Barcelona, Spain

Publisher Item Identifier S 0278-0046(01)03380-9. constrained to track the reference by means of the control action.

Several tracking control schemes have been suggested for dc-to-ac conversion, depending on which processing is applied to the signal error. For instance, many tracking control techniques based on high-frequency pulsewidth modulation (PWM) have been proposed for buck-based inverters [1]-[5]. In all of these cases, the control design is based on a power-stage model, leading to output waveforms sensitive to power-stage parameter variations, such as the output load.

On the other hand, sliding-mode control techniques have been proposed as an alternative to PWM control strategies in dc-dc switching regulators since they make these systems highly robust to perturbations, namely, variations of the input voltage and/or in the load [6]-[11]. For this reason, tracking control schemes based on these techniques have also been applied to the design of high-efficiency buck-based inverters. In this case, the converter output is forced to track an external sinusoidal reference by means of an appropriate sliding-mode control action [12]-[19]. It will be shown in the next section that the existing sliding-mode tracking control schemes present some drawbacks in the design phase, such as analytical difficulties in establishing the tradeoff between the transient state period and the sliding domain where the sliding motion is ensured [14]. As a result, no design procedure leading to the appropriate control action can be easily derived.

These tracking control schemes have also been employed in applications where an external sinusoidal reference is not inherently required, as in the case of ac power sources. The ac power sources, generally used in testing electronic equipments which operate with ac power input signals (for instance, supplied by single-phase mains), are designed in order to achieve both programmable amplitude and frequency of the output signal. Consequently, the design of such systems based on a tracking control scheme will also require the design of a low-power programmable sinusoidal generator acting as a reference signal.

As an alternative, and starting from a sliding control tracking scheme, this work presents a methodology leading to a sliding control law for ac signal generation in the buck converter, where no external signal reference is needed. Accordingly, the requirement of a low-power reference signal in the control loop is removed. Due to the absence of a reference signal this autonomous control policy will be referred to as a "generation" control scheme.

Besides exhibiting the robustness properties with respect to parameter variations of the sliding control technique, the proposed "generation" control law leads to a zeroth-order dynamics 
in sliding motion in contrast to sliding control techniques based on tracking schemes. Furthermore, a design procedure ensuring the sliding regime can be easily obtained. All these features will be evidenced in the following sections.

The paper is organized as follows. Section II focuses on the characteristics of existing sliding control laws leading to the tracking of an arbitrary signal at the buck converter output. Subsequently, Section III introduces a methodology to obtain a generating control scheme from a tracking one. Section IV applies this methodology for the case of sinusoidal generation in a buck converter with resistive load. As a result, a switching surface, a sliding control law, and a set of design restrictions in terms of the converter components and the parameters of the desired sinusoidal output signal are obtained. These results are then generalized to the cases of reactive and nonlinear loads. Additionally, the influence of the converter resistive losses is also analyzed. In Section V, numerical simulations showing the robustness of the proposed control system and experimental results of a laboratory prototype are presented to illustrate the application of the method. Finally, Section VI summarizes the conclusions of the work.

\section{Previous Considerations ON Sliding-Mode SigNAL TRACKING}

Switching power converters constitute a natural field of application for the sliding-mode control technique according to the abrupt topological changes that the circuit commanded by a discontinuous control action undergoes. The main steps of this control policy can be summarized, by using the equivalent control concept, as follows [6], [8].

- The first step is the choice of a switching surface $\sigma(x, t)=0$ (where $x$ is the system state vector) that provides the desired asymptotic behavior in steady state.

- Obtaining the equivalent control $u_{e q}$ by applying the invariance condition

$$
\left.\frac{d \sigma}{d t}\right|_{\substack{\sigma=0 \\ u=u_{e q}}}=0
$$

constitutes the second step. The existence of the equivalent control $u_{e q}$ assures the feasibility of a sliding motion over the switching surface $\sigma(x, t)=0$. On the other hand, beside describing the averaged dynamical behavior of the power stage over the switching surface, the equivalent control enables obtaining the sliding domain, given by

$$
\min \left\{u^{-}, u^{+}\right\}<u_{e q}<\max \left\{u^{-}, u^{+}\right\}
$$

where $u^{-}$and $u^{+}$are the control values for $\sigma<0$ and $\sigma>0$ respectively. The sliding domain is the state plane region where the sliding motion is ensured.

- Finally, the control law is obtained by guaranteeing the Lyapunov stability criteria, i.e., $d \sigma^{2} / d t<0$.

The first problem considered in this work focuses on how the output voltage $v(t)$ of the full-bridge buck inverter, depicted in Fig. 1, can be forced to track an arbitrary function $f(t)$, by applying the sliding-mode control technique.

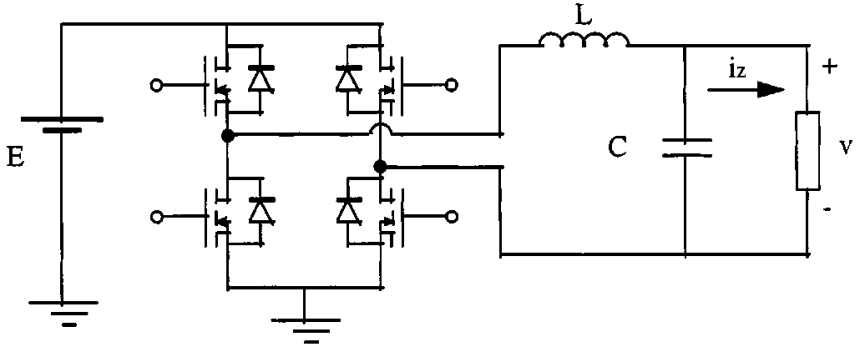

Fig. 1. Full-bridge buck inverter.

Considering a resistive load, the power stage can be represented by the following set of differential state equations, where $i$ and $v$ designate the inductor current and the capacitor voltage, respectively:

$$
\frac{d}{d t}\left[\begin{array}{c}
i \\
v
\end{array}\right]=\left[\begin{array}{cc}
0 & -\frac{1}{L} \\
\frac{1}{C} & -\frac{1}{R C}
\end{array}\right] \cdot\left[\begin{array}{l}
i \\
v
\end{array}\right]+\left[\begin{array}{c}
E / L \\
0
\end{array}\right] \cdot u
$$

where $u$ stands for the control input, taking values in the discrete set $\{-1,1\}$ depending on which switches are active, namely,

$$
\begin{aligned}
& u=-1 \text { corresponds to input filter voltage equal to }-E \\
& u=+1 \text { corresponds to input filter voltage equal to }+E .
\end{aligned}
$$

As it has been shown by Carpita et al. in [13], [14], the following switching surface leads to a robust output voltage behavior with respect to load variations and source perturbations, due to its independence on converter parameters:

$$
\sigma_{c}(t)=\lambda \cdot e(t)+\frac{d e(t)}{d t}=0
$$

where $e(t)$ stands for the voltage error defined as $e(t)=v(t)-$ $f(t), v(t)$ being the output voltage and $f(t)$ the voltage reference signal.

The sliding motion over the switching surface provides a firstorder dynamic transient response leading to the desired steadystate behavior, that is, :

$e(t)=e(0) \cdot e^{-\lambda \cdot t}$
$v(t)=e(0) \cdot e^{-\lambda \cdot t}+f(t) \stackrel{t \rightarrow \infty}{\longrightarrow} e(t) \rightarrow 0 \Rightarrow v(t)=f(t)$

this meaning a direct tracking of the reference signal $f(t)$ of the output voltage $v(t)$.

The corresponding equivalent control resulting from the application of the invariance condition to $\sigma_{c}$ is given by

$$
\begin{aligned}
\frac{d \sigma_{c}(t)}{d t} & =0 \Rightarrow \text { ueq }(t) \\
& =\frac{L C}{E} \cdot\left[\frac{d^{2} f(t)}{d t^{2}}+\lambda \cdot \frac{d f(t)}{d t}+\left(\frac{1}{R C}-\lambda\right) \cdot \frac{d v}{d t}+\frac{v}{L C}\right]
\end{aligned}
$$

or, by replacing (3) in the previous expression,

$$
\begin{aligned}
& u e q(t)=\frac{L C}{E} \cdot\left[\frac{d^{2} f(t)}{d t^{2}}+\frac{1}{R C} \cdot \frac{d f(t)}{d t}+\frac{1}{L C} \cdot f(t)\right. \\
& \left.+\left(\lambda^{2}-\frac{\lambda}{R C}+\frac{1}{L C}\right) \cdot e(0) \cdot e^{-\lambda t}\right]
\end{aligned}
$$


whereas the sliding domain can be obtained by imposing

$$
\begin{aligned}
\min \left\{u^{-}, u^{+}\right\}<u_{e q} & <\max \left\{u^{-}, u^{+}\right\}, \\
& \text {or equivalently }-1<u_{e q}<1
\end{aligned}
$$

this leading to restrictions among the values of the converter parameters $E, L, C, R$, the desired output signal $f(t)$, and the desired transient dynamics represented by the time constant $1 / \lambda$. These restrictions have to be taken into account in the design procedure.

As can be seen from (3), the constant $\lambda$, for a fast transient response, has to be selected as large as possible. However, as is stated in [14], the greater the value of $\lambda$ is, the faster the transient response, but the greater the equivalent control value is. As a consequence, the sliding motion over $\sigma_{c}$ can be lost due to the bounds on control.

On the other hand, the maximum and the minimum values of the equivalent control required to test when the system dynamics escapes from the switching surface can not be easily deduced from (4), due to both the analytical complexity and the dependence on the value of the initial error $e(0)$ (i.e., dependence on the initial conditions). As a result, no design procedure ensuring the sliding motion can be easily established.

When the application does not require an external reference, the work reported here proposes an autonomous (time independent) switching surface leading to the same steady-state behavior, which can be derived from the surface defined in (2) by using the inverse function theorem. As will be shown in the following sections, by applying this method a zeroth-order dynamics is expected in sliding motion. Furthermore, a useful design procedure can be easily derived.

\section{From Tracking to GenERATION}

An autonomous control scheme (time independent) can be obtained from a time-dependent one by expressing the switching surface (2) only in terms of the power-stage state variables, therefore removing the need of an external time-variable reference [20]. This can be done by noticing that the time variable, $t$ in (2), can be locally written in terms of the state variable $v$ by using the inverse function $f^{-1}$ of $f(t)$ (supposed continuous and derivable), as follows:

$$
\begin{aligned}
& \text { If } v=f(t) \quad \forall t_{0} \text { such that }\left.\frac{d f(t)}{d t}\right|_{t=t_{o}} \neq 0 \\
& \text { then } t=f^{-1}(v) \text { in a neighborhood of } t_{0} .
\end{aligned}
$$

By replacing $t=f^{-1}(v)$ in (2), the following autonomous (time independent) switching surface $\sigma_{s}$ is obtained:

$$
\sigma_{s}(v)=\lambda \cdot\left(v-f\left(f^{-1}(v)\right)\right)+\left[\frac{d v}{d t}-\left.\frac{d f}{d t}\right|_{t=f^{-1}(v)}\right]=0
$$

or, equivalently,

$\sigma_{s}\left(v, \frac{d v}{d t}\right)=\left[\frac{d v}{d t}-\left.\frac{d f}{d t}\right|_{t=f^{-1}(v)}\right]=\left.\frac{d e(t)}{d t}\right|_{t=f^{-1}(v)}=0$.

This last expression highlights that the output voltage desired behavior can be reached by defining an autonomous switching surface, which is independent of the load value, and sensing both the output voltage and the capacitor current of the power stage.

Notice that the inverse function theorem has been applied in the case that the ideal sliding dynamics, given by (3), correspond to the steady state, i.e., $v=f(t)$, yielding a steady-state differential relation between $v$ and $\dot{v}$. In the autonomous formulation (from the generation point of view) the previous steady-state differential relation holds when the system trajectory reaches and remains on the sliding surface, by means of a proper control action. In this sense, a zeroth-order dynamics in sliding motion is expected, this constituting one of the main differences with respect to the original sliding-mode control proposed in [13] and [14].

The usefulness of this procedure stems from the fact that, given a desired output voltage $v(t)=f(t)$, a time-invariant, load-independent, and source-independent switching surface with zeroth-order sliding motion dynamics (this meaning no transient response in sliding motion) can be designed by applying (7), provided that on one hand, $f(t)$ satisfies the requirements of the inverse function theorem and, on the other hand, the inverse function $f^{-1}$ can be described by means of an analytical expression. In practical cases the requirement given by (5) is not verified for all $t_{0}$, as in the case of periodical signals. However, the method can be applied disregarding this requirement and modifying the resulting control law (and then the sliding surface) for the points such that $(d f / d t)=0$. The case of sinusoidal signal, exposed in the following sections, will illustrate this procedure.

\section{SinUsoidal Voltage Generation}

\section{A. Switching Surface and Sliding Domain}

The general procedure presented in the previous section can be applied to obtain an autonomous switching surface for generating the sinusoidal output voltage

$$
v(t)=f(t)=A \sin (\omega t)+B \text {. }
$$

In this case, $t=f^{-1}(v)=(1 / \omega) \arcsin ((v-B) / A)$, so (7) becomes

$$
\sigma_{s}(v, \dot{v})=\dot{v}-A \omega \cos \left[\arcsin \left(\frac{v-B}{A}\right)\right]=0
$$

which can be simplified, by using trigonometric properties, as

$$
\sigma_{s}(v, \dot{v})=\left[\dot{v} \mp A \omega \sqrt{1-\left(\frac{v-B}{A}\right)^{2}}\right]=0
$$

where the - and + signs apply when $\dot{v}>0$ and $\dot{v}<0$, respectively. Equation (9) can also be expressed as

$$
\sigma(v, \dot{v})=\dot{v}^{2}+\omega^{2} \cdot(v-B)^{2}-\omega^{2} \cdot A^{2}=0 .
$$

Equation (10) corresponds to the canonical expression of an ellipse in the $(v, \dot{v})$ plane centered on $(B, 0)$ and with vertical and horizontal semiaxis $A \omega$ and $A$, respectively (see Fig. 2). Therefore, by choosing the autonomous switching surface (10), the desired ac output will be obtained over the surface $\sigma(v, \dot{v})=0$. 


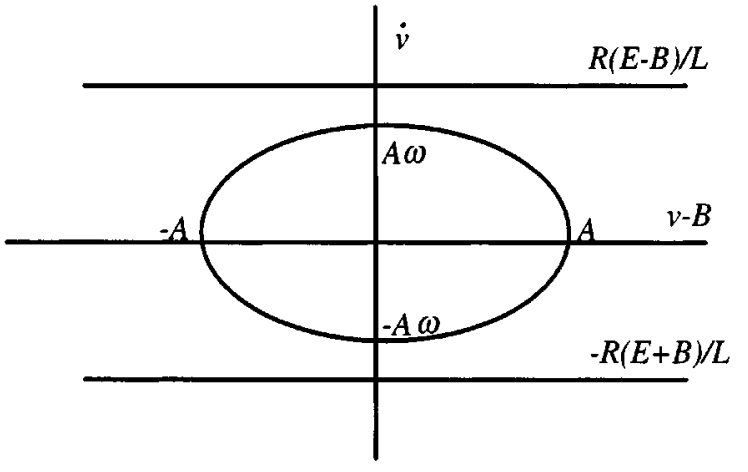

Fig. 2. Sliding surface and sliding domain in the phase plane for the case of a generated frequency $\omega=1 / \sqrt{L C}$.

By applying the invariance condition to (10),

$$
\frac{d \sigma}{d t}=2 \ddot{v} \ddot{v}+2 \omega^{2} \dot{v}(v-B)=0 \Rightarrow\left\{\begin{array}{l}
\dot{v}=0 \\
\text { or } \\
\ddot{v}+\omega^{2}(v-B)=0 .
\end{array}\right.
$$

From (11) and (1), the equivalent control $u_{e q}$ can be obtained in terms of $v$ and $\dot{v}$ as follows:

$$
E \cdot u_{e q}=\frac{L}{R} \dot{v}+\left(1-L C \omega^{2}\right) \cdot(v-B)+B .
$$

Finally, the sliding domain is given by (10) and

$$
-E<\frac{L}{R} \dot{v}+\left(1-L C \omega^{2}\right) \cdot(v-B)+B<E .
$$

Note that a proper control leading to a sliding motion over any other switching surface (obtained by modifying the parameters $A, B$, and $\omega$ ) can be designed provided that this surface is located in the region of the plane $(v, \dot{v})$ bounded by two straight lines $r_{1}, r_{2}$ given by

$$
\left\{\begin{array}{l}
r_{1}: \frac{L}{R} \dot{v}+\left(1-L C \omega^{2}\right) \cdot(v-B)+B=E \\
r_{2}: \frac{L}{R} \dot{v}+\left(1-L C \omega^{2}\right) \cdot(v-B)+B=-E
\end{array}\right.
$$

Fig. 2 shows these boundaries in the case where the frequency $\omega$ of the generated signal is equal to the characteristic frequency $1 / \sqrt{L C}$ of the converter output filter.

\section{B. Design Restrictions}

The previous section has established the sliding domain, which imposes a set of design restrictions in terms of both the converter and the output signal parameters. In contrast to the case of the tracking scheme exposed in Section II, these restrictions can be easily derived, leading to a useful design procedure. Replacing the expression of the desired output voltage (8) in the previous inequality (13) leads to

$-E<\frac{L}{R} \cdot \omega \cdot A \cos (\omega t)+\left(1-L C \omega^{2}\right) \cdot A \sin (\omega t)+B<E \quad \forall t$

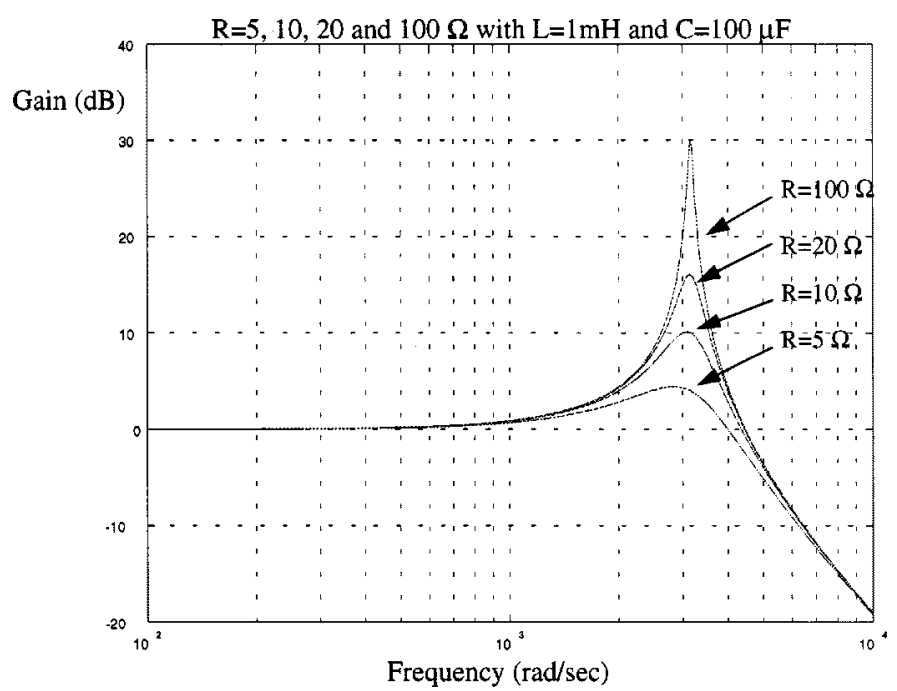

Fig. 3. Plot of $(A / E)_{\max }$ versus output signal frequency.

or, equivalently

$$
A<\frac{E-|B|}{L C} \cdot \frac{1}{\sqrt{\frac{\omega^{2}}{(R C)^{2}}+\left(\omega^{2}-\frac{1}{L C}\right)^{2}}} .
$$

In the case of a dc-ac conversion $(B=0)$, the previous restriction can be reduced to

$$
A<E \cdot \gamma(\omega)
$$

where

$$
\gamma(\omega)=\frac{1}{L C} \cdot \frac{1}{\sqrt{\frac{\omega^{2}}{(R C)^{2}}+\left(\omega^{2}-\frac{1}{L C}\right)^{2}}}
$$

Fig. 3 shows the plot of the boundary $(A / E)_{\max }$ or, equivalently, $\gamma(\omega)$ versus the output signal frequency, taking the load value $R$ as a parameter.

As a result, the sliding domain can be expressed as a function of the frequency response of the converter output filter and as a function of the output signal parameters (amplitude, offset and frequency). This suggests the following design procedure.

When an output signal without offset is desired, that is, $B=$ 0 , given a value of $R$, the sliding regime is ensured for the values of the output amplitude-input voltage ratio $(A / E)$ lying below the plot of the output filter frequency response. On the other hand, given an output amplitude for which the sliding motion exists, the sliding motion is also ensured when the output load is increased. For this reason, the design has to take into account the minimum load value for which a sinusoidal output signal is desired. Moreover, an amplitude of the output signal greater than the dc input voltage can be obtained for a small frequency range in the vicinity of the output filter resonant frequency. Furthermore, a large frequency range of output signal can be obtained with output amplitude lower than the input voltage corresponding to the flat zone of the curve. 
1) General Load Case: This previous study is not restricted to resistive loads and can be generalized to reactive or nonlinear loads. Starting from the scheme of Fig. 1, where a generic load is characterized by means of the output current $i_{Z}$, it can be written that

$$
\begin{aligned}
& \frac{d i}{d t}=\frac{1}{L}(E \cdot u-v) \\
& \frac{d v}{d t}=\frac{1}{C}\left(i-i_{Z}\right) .
\end{aligned}
$$

By applying the same relationships as in the case of resistive loads, the expression for the equivalent control will be given by

$$
u_{e q}=\frac{\frac{1}{C} \cdot \frac{d i_{Z}}{d t}+\frac{1}{L C} \cdot v-\omega^{2}(v-B)}{\frac{1}{L C} \cdot E}
$$

whereas the sliding domain corresponds to the following expression:

$$
\left|L \frac{d i_{Z}}{d t}+B\right|<|E-| 1-L C \omega^{2}|\cdot A| .
$$

It can be pointed out that the system trajectory escapes from the sliding surface when the load has current discontinuities. In this case, the control policy has to be designed in order to recover the desired sliding motion.

2) Reactive Load Case: When the load has a reactive component, (18) becomes

$$
u_{e q}(s)=\frac{V(s)}{E} \cdot \frac{L \cdot s+\left(1-\omega^{2} L C\right) \cdot Z_{i}(s)}{Z_{i}(s)}+\frac{B \omega^{2} L C}{E}
$$

where $Z_{i}(s)$ is the load impedance, defined by

$$
Z_{i}(s)=\frac{V(s)}{I_{Z}(s)}
$$

where $V(s)$ and $I_{Z}(s)$ stand for the Laplace transforms of $v(t)$ and $i_{Z}(t)$, respectively.

In order to obtain the sliding domain and taking into account that $v(t)=A \sin (\omega t)+B$, the equivalent control is given by

$$
u_{e q}(t)=\frac{A}{E} \cdot|G(j \omega)| \cdot \sin (\omega t+\angle G(j \omega))+\frac{B}{E}
$$

where $|G(j \omega)|$ and $\angle G(j \omega)$ are the gain and the phase, respectively, of the output filter transfer function loaded with the reactive load:

$$
G(s)=\frac{L \cdot s+\left(1-\omega^{2} L C\right) \cdot Z i(s)}{Z i(s)} .
$$

The sliding domain will be obtained as in previous cases, by imposing $-1<u_{e q}<1$, this leading to

$$
A<\frac{(E-|B|) \cdot\left|Z_{i}(j \omega)\right|}{\left|Z_{i}(j \omega) \cdot\left(1-L C \omega^{2}\right)+j L \omega\right|} .
$$

This expression suggests that the same design procedure exposed above can also be applied to reactive loads by using the Bode diagram of the output filter loaded with the reactive impedance.

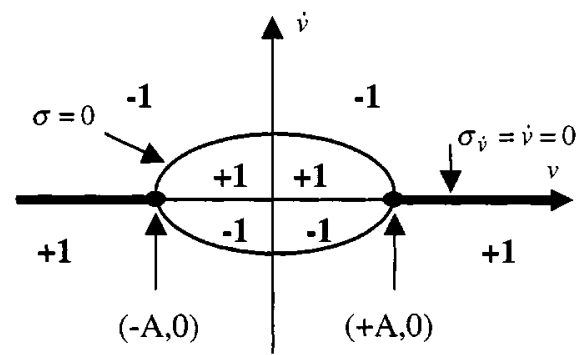

Fig. 4. Values of the control variable $u$ in the $(v, \dot{v})$ plane. Thick lines, undesired sliding surface.

\section{Switching Control Law}

Accounting for sliding-mode control principles, the power converter can reach the switching surface $\sigma$ if the control action guarantees Lyapunov stability criteria. Therefore, from (10),

$$
\frac{d \sigma^{2}}{d t}=2 \sigma \frac{d \sigma}{d t}=2 \sigma 2 \dot{v}\left(\ddot{v}+\omega^{2}(v-B)\right)
$$

and according to the state-space equations given in (1), the previous expression can be rewritten as

$$
\frac{d \sigma^{2}}{d t}=4 \sigma \dot{v}\left(\frac{E}{L C} u-\left(\frac{1}{R C} \dot{v}+\left(\frac{1}{L C}-\omega^{2}\right) \cdot v+\omega^{2} B\right)\right) .
$$

Taking into account the expression of $u_{e q}$ given by (12) and applying the invariance condition, (23) can be simplified to

$$
\frac{d \sigma^{2}}{d t}=4 \sigma \cdot \dot{v}\left[\frac{E}{L C}\left(u-u_{e q}\right)\right]
$$

so that, recalling that $-1<u_{e q}<1$, the previous expression leads to the following control law:

$$
\begin{aligned}
& \text { if } \sigma \cdot \dot{v}>0, \quad \text { then } u<u_{e q}, \text { therefore } u=-1 \\
& \text { if } \sigma \cdot \dot{v}<0, \quad \text { then } u>u_{e q}, \text { therefore } u=+1 .
\end{aligned}
$$

Fig. 4 shows the value of the control variable in the $(v, \dot{v})$ plane. Two switching surfaces are involved in this control policy, namely, the following:

1) $\sigma=0$, which is the designed sliding surface leading to the desired output behavior;

2) $\dot{v}=0$, which will be referred as $\sigma_{i}=\dot{v}=0$ and corresponds to the curve of the equilibrium points of the buck power stage.

Unfortunately, the control law defined in (25) and (26) leads also to a sliding motion over this later switching surface $\sigma_{i}=$ $\dot{v}=0$ in the regions located outside the ellipse. As a consequence, if this switching surface is reached, the system will remain on the classic regulation operation mode $(\dot{v}=0)$ and will deliver a constant output voltage. Furthermore, even in the case that the system would reach first the desired sliding surface $\sigma=0$, it would finally operate in regulation mode since the sliding surface $\sigma=0$ intersects $\sigma_{i}=0$ on the $v$ axis at the points $(-A, 0)$ and $(+A, 0)$ as is depicted in Fig. 4. This undesired behavior can be avoided by modifying the control action in a region of a small width $k$ near the equilibrium curve 


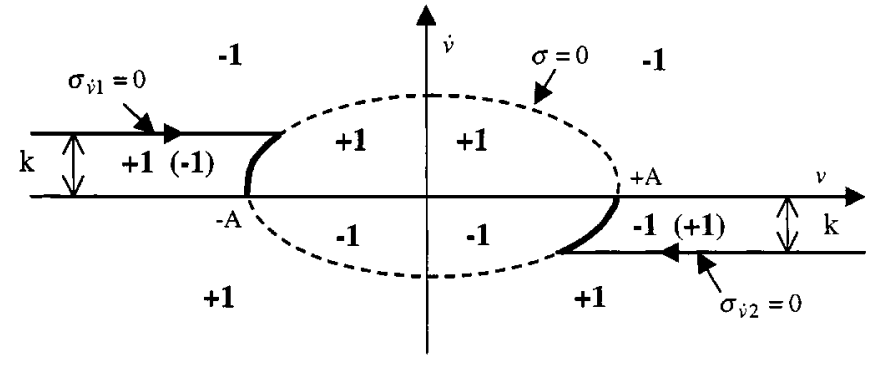

Fig. 5. Control value and additional sliding surfaces $\sigma_{1 i}$ and $\sigma_{2 i}$. Thick lines: sliding motion loss regions over the desired sliding surface $\sigma=0$. Between brackets, previous control variable values according to (25) and (26).

$\sigma_{\dot{v}}=0$ when $\sigma>0$. One possible choice, regarding the required simplicity and robustness of a future implementation, is to introduce two additional switching surfaces, denoted as $\sigma_{1 i}$ and $\sigma_{2 i}$ such that

$$
\begin{array}{ll}
\sigma_{1 \dot{v}}=\dot{v}-k, & (k>0) \text { for } \dot{v}>0 \text { and } \sigma>0 \\
\sigma_{2 \dot{v}}=\dot{v}+k, & (k>0) \text { for } \dot{v}<0 \text { and } \sigma>0 .
\end{array}
$$

Fig. 5 shows both the control value and the additional switching surfaces $\sigma_{1 i}$ and $\sigma_{2 i}$ in the $(v, \dot{v})$ plane and will be referred to as the "modified control law."

It can be easily shown that the control law depicted in Fig. 5 also leads to a sliding motion over these two additional switching surfaces, where the ideal sliding dynamics are given by

$$
\begin{gathered}
\text { For } \sigma_{1 i}=0 \begin{array}{c}
v(t)=k\left(t-t_{0}\right)+v\left(t_{0}\right), \\
(k>0) \text { thus } v(t) \text { increases with time }
\end{array} \\
\text { For } \sigma_{2 i}=0 \begin{array}{c}
v(t)=-k\left(t-t_{0}\right)+v\left(t_{0}\right), \\
(k>0) \text { thus } v(t) \text { decreases with time }
\end{array}
\end{gathered}
$$

therefore, in each case the system will evolve, as shown by the arrows in Fig. 5, toward the desired sliding surface $\sigma=0$; the equivalent control, for resistive loads, corresponds to the following expression:

$$
u_{e q i}=\frac{E-\frac{L}{R}\left(\frac{v}{R C}-\frac{i}{C}\right)}{v} .
$$

It can be pointed out that the modified control variable value is different from the first one defined in (25) and (26) in the regions outside the ellipse comprised between $\dot{v}=0$ and $\dot{v}=$ $\pm k$, as depicted in Fig. 5. Consequently, there results a certain amount of distortion in the sinusoidal output voltage, due to a loss of sliding motion over the desired surface $\sigma=0$.

The distortion due to the modification of the switching control law can be controlled by a proper design of the $k$ parameter. The final value of $k$ is easily adjusted experimentally in order to satisfy a previously fixed distortion index. Anyway, this final value presents a tradeoff: the greater the value of $k$, the greater the sliding motion loss region, but according to (29) and (30), the faster the system recovering to the desired sliding surface.

The proposed procedure can also be applied for a switching scheme $u=+1,0,-1$. However, in this case, the analysis leads to a control value $u$ that depends on the equivalent control. Therefore, the control law depends on the load value.

\section{Influence of the Converter Losses in the Design}

This section analyzes, as in [14] and [19], whether the previous switching surface and control law can be applied to output voltage sinusoidal generation when the converter resistive losses are taken into account. The application of the previous method in the buck inverter with series resistive losses of the inductance $\left(r_{L}\right)$ and the capacitor $\left(r_{C}\right)$ leads to the following autonomous switching surface:

$\sigma(v, \dot{v}):=\dot{v}^{2}+\omega^{2} \cdot(v-B)^{2}-\omega^{2} \cdot\left(\frac{A}{\sqrt{1+\left(r_{C} C \omega\right)^{2}}}\right)^{2}=0$

where $v(t)$ is the capacitor voltage when the desired output voltage is $v_{0}(t)=A \sin (\omega t)+B$. On the other hand, the sliding control law is given by (25) and (26) as well.

As can be seen, in the case of converter losses, the desired sinusoidal output voltage with amplitude $A$ can be obtained by using the original switching surface defined in (10) and rewritten here for convenience

$$
\sigma(v, \dot{v}):=\dot{v}^{2}+\omega^{2} \cdot(v-B)^{2}-\omega^{2} \cdot A^{2}=0
$$

if $A$ can be modified to $A / \sqrt{1+\left(r_{C} C \omega\right)^{2}}$. As will be shown in the following section, this modification can be easily performed by means of an external amplitude tuning, which is a common procedure in any practical implementation due to the influence of nonidealities.

Finally, it can be pointed out that the sliding domain is also dependent on the converter losses. It can be proved that the aforementioned design procedure for a converter with no losses can be applied in this case by considering the Bode plot of the output filter including the inductance and capacitor resistance losses.

\section{Simulation AND ExPERIMENTAL RESUlts}

Due to the nonlinearity of the sliding surface and the phase-plane partition leading to the modified control law described in Section IV, the control loop has been implemented through a lookup table recorded on an electrically programmable read-only memory (EPROM) device. As an alternative, the design of a microelectronic BiCMOS analog circuit intended to implement the sliding-mode controller is currently in progress. The proposal of that analog application-specific integrated circuit (ASIC) design, which operates in current-mode signal processing, represents good performance as far as operation speed, power consumption, suitability for low-voltage operation, and interference robustness are concerned. HSPICE transistor-level simulations for a BiCMOS $1.2-\mu \mathrm{m}$ technology validate the functionality of the proposed sliding-mode controller implementation for switching frequencies up to $1 \mathrm{MHz}$. Complete design details at the microelectronic analog design level have been recently proposed and described in [21].

The EPROM-based control loop scheme is depicted in Fig. 6. The main objective of the EPROM device is to deliver the control value $(u)$ from the values of $v$ and $\dot{v}$. Therefore, the EPROM 


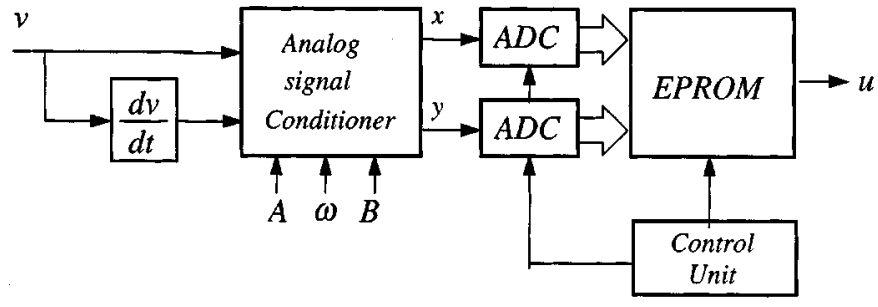

Fig. 6. Control loop scheme.

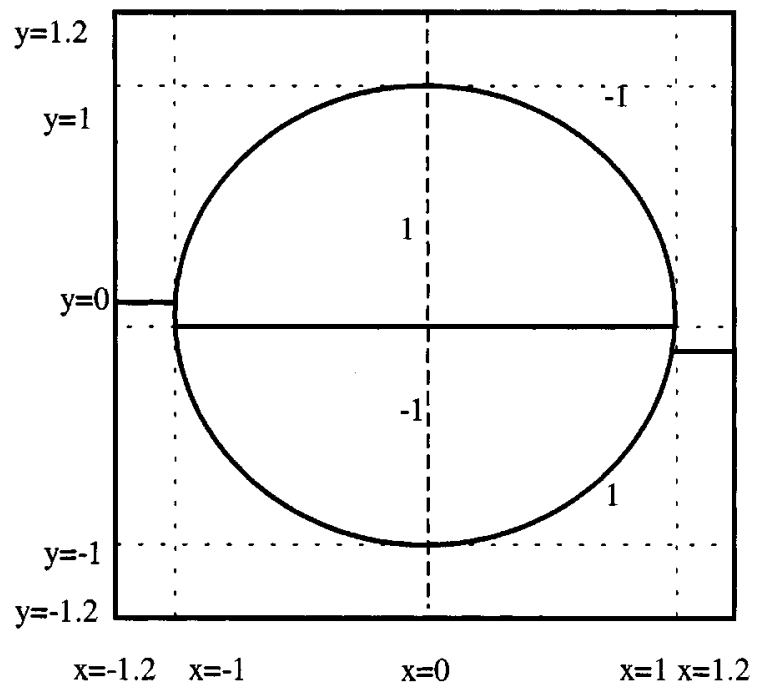

Fig. 7. $X Y$ phase plane digitized in the EPROM.

device is only recorded with the $u$ values map $(u=+1$ or -1$)$ shown in Fig. 5.

In order to record the EPROM only once, whatever the desired sinusoidal output is (in terms of the parameters $A, B$ and $\omega$ ), the variables $v$ and $\dot{v}$ of the buck converter are scaled according to

$$
y=\frac{1}{A \omega} \cdot \frac{d v}{d t} \quad x=\frac{(v-B)}{A} .
$$

Hence, the switching surface with the new variables defined by these previous equations becomes

$$
\sigma(x, y)=x^{2}+y^{2}-1=0
$$

which corresponds to a unit circle in the normalized $X Y$ plane. This change of variables can be performed by means of an externally programmable signal conditioner implemented through standard analog circuitry. It should be noted that, with this procedure, the sinusoidal output signal parameters can be selected by modifying dc voltage values of $A, B$, and $\omega$, removing the requirement of an external programmable reference oscillator.

The control law values, taking into account the expected dynamic range of the variables $x$ and $y$, are shown in the phase plane in Fig. 7. From the digitized pairs $(x, y)$, the EPROM output gives the value of the control variable $u$ according to Fig. 7.

The previous control law has been simulated for a buck converter with the following parameters: $L=1 \mathrm{mH}, C=100 \mu \mathrm{F}$, and $E=12 \mathrm{~V}$. The digital control loop has been modeled in

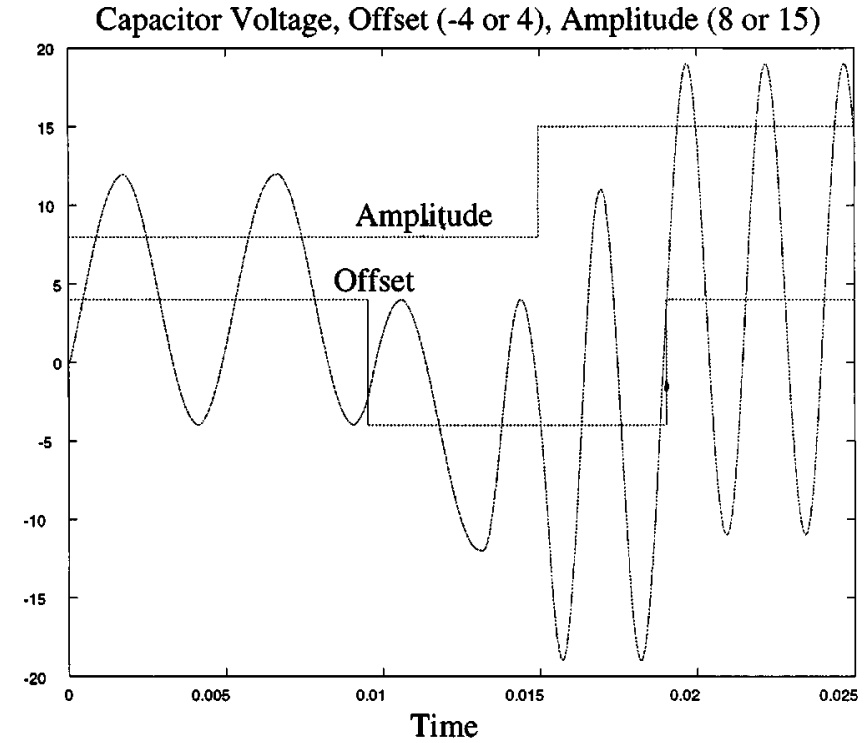

Fig. 8. Output voltage simulation for different values of $R, A, B$, and $\omega$.

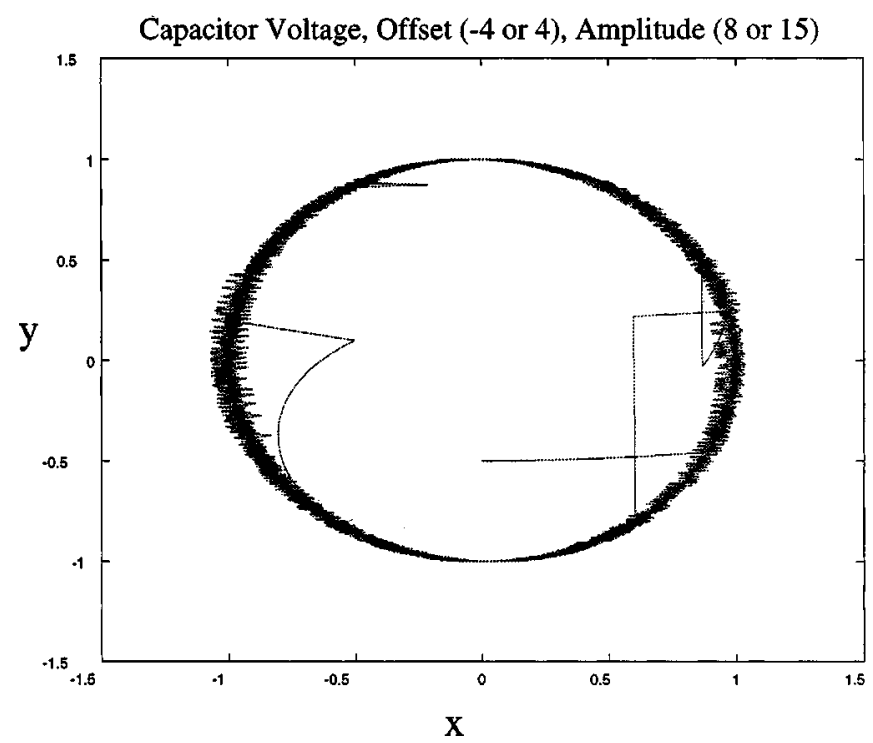

Fig. 9. Normalized $X Y$ phase-plane simulation.

the simulation by including a sample-and-hold operating at 300 $\mathrm{kHz}$ and assigning 8 bits to the normalized output voltage and 12 bits to the normalized output voltage derivative. Figs. 8 and 9 show the closed-loop system response and the normalized phase plane $X Y$ simulations for different values of the output signal parameters $A, B$ and $\omega$, when the load has a reactive component of $L=2 \mathrm{mH}$. The amplitude has been changed from 8 to $15 \mathrm{~V}$ and the frequency from 200 to $400 \mathrm{~Hz}$ at $t=0.0135 \mathrm{~s}$, whereas the offset has been changed from -4 to $4 \mathrm{~V}$ and the converter has been loaded with a pulsating load varying between $10-100 \Omega$ at a rate of $450 \mathrm{rad} / \mathrm{s}$. As can be seen, the output voltage reaches the desired steady-state behavior (according to the sinusoid parameter changes) with fast transient response and no load influence.

In order to experimentally verify the proposed sliding-mode control, an electronic prototype has been implemented. The input voltage and the resistive load are fixed to $E=12 \mathrm{~V}$ and $R=5 \Omega$, respectively, and the desired output signal is 


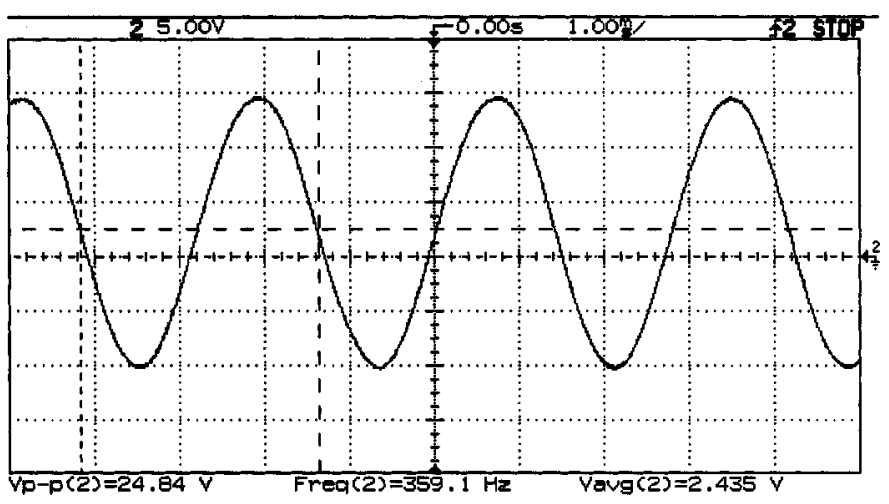

Fig. 10. Experimental output voltage.

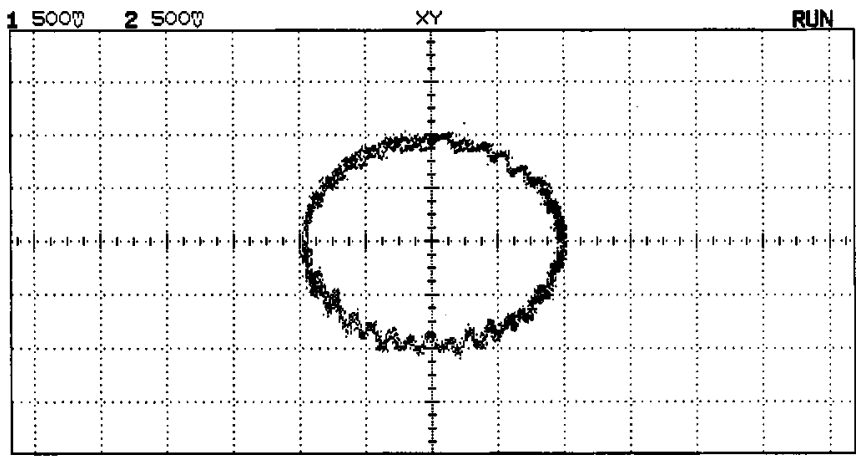

Fig. 11. Experimental $X Y$ phase plane.

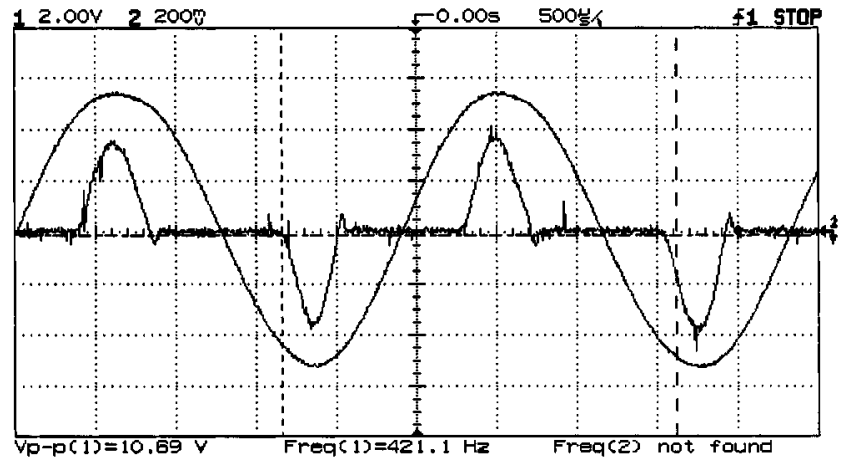

Fig. 12. Measured output voltage and output current when the buck converter is loaded with a full-wave rectifier.

$v_{0}(t)=12 \cdot \sin (2 \pi 350 t)$, this meaning that the output amplitude-input voltage ratio is $(A / E)=1$. The buck converter parameter values $L=1 \mathrm{mH}$ and $C=100 \mu \mathrm{F}$ have been chosen in order to remain in the sliding domain defined by (15), since $(A / E)_{\max } \approx 1.4$. The sampling frequency is fixed at $300 \mathrm{kHz}$, that is, $10 \times$ the expected maximum switching frequency. Fig. 10 shows the experimental output voltage in this case. The phase plane $X Y$ is shown in Fig. 11 for a desired output amplitude of $9 \mathrm{~V}$. Finally, in order to check the robustness of the design, a full-wave rectifier has been inserted between the converter and the resistive load. In this case, the desired output signal is given by $v_{0}(t)=5.35 \cdot \sin (2 \pi 420 t)$, and the converter parameters are the same as in the previous case. The measured output voltage and output current are shown in Fig. 12. The behavior for a high power level will constitute the objective of further research.

\section{CONCLUSIONS}

In this paper, a sliding-mode control scheme with no external reference for a buck-based sinusoidal inverter, with programmable amplitude and frequency, has been proposed. Starting from robust (to both load and source perturbations) sliding control laws for tracking of a desired reference at the buck converter output, a general procedure based on the application of the inverse function theorem has been established. The new procedure removes the requirement of a reference signal, leading to a signal generation control scheme. As a result, an autonomous (time independent) switching surface and its corresponding robust sliding control law are obtained. This general procedure has been applied to the particular case of sinusoidal generation. In the generation control scheme, zeroth-order dynamics in sliding motion are obtained, constituting the main difference with tracking control techniques. On the other hand, a set of design restrictions arising from the sliding-domain analysis has been established. This analysis has been completed taking into account the converter losses and considering both resistive and reactive loads. In all cases, these design restrictions can be expressed as a function of the output signal parameters (amplitude and frequency) and the buck-based inverter components in terms of the output filter Bode diagram, this facilitating the subsequent design procedure.

Finally, due to the nonlinearity of the resulting sliding surface, the control loop has been implemented through a lookup table recorded on an EPROM device. Nevertheless, the design of a microelectronic BiCMOS analog circuit intended to implement the sliding-mode controller is currently in progress. The theoretical predictions have been validated by means of simulations and experimental results for both linear and nonlinear loads.

\section{ACKNOWLEDGMENT}

The authors would like to thank A. Jiménez Marquez, J. Gavaldá Ferré, and X. Díaz García their help with the experimental results of this work.

\section{REFERENCES}

[1] A. Capel, J. C. Marpinard, J. Jalade, and M. Valentin, "Large-signal dynamic stability analysis of synchronized current-controlled modulators-Application to sine-wave power inverters," ESA J., vol. 7, pp. 63-74, 1983.

[2] A. Kawamura and R. G. Hoft, "Instantaneous feedback controlled PWM inverter with adaptative hysteresis," IEEE Trans. Ind. Applicat., vol. IA-20, pp. 706-712, July/Aug. 1984.

[3] K. P. Gokale, A. Kawamura, and R. G. Hoft, "Dead-beat microprocessor control of PWM inverter for sinusoidal output waveform synthesis," IEEE Trans. Ind. Applicat., vol. IA-23, pp. 901-910, Sept./Oct. 1985.

[4] P. Maussion, M. Grandpierre, J. Faucher, and J. C. Hapiot, "Instantaneous feedback control of a single-phase PWM inverter with nonlinear loads by sine wave tracking," in Proc. IEEE IECON'89, 1989, pp. 130-135.

[5] K. Jezernik, M. Milanovic, and D. Zadravec, "Microprocessor control of PWM inverter for sinusoidal output," in Proc. EPE'89, 1989, pp. 47-51.

[6] V. I. Utkin, Sliding Mode and Their Applications in Variable Structure Systems. Moscow, U.S.S.R.: Mir, 1978.

[7] F. Bilanovic, O. Music, and A. Sabanovic, "Buck converter regulator operating in the sliding mode," in Proc. 7th Int. PCI Conf. Power Conversion, 1983, pp. 251-258. 
[8] H. Sira-Ramirez, "Sliding motions in bilinear switched networks," IEEE Trans. Circuits Syst., vol. CAS-34, pp. 919-933, Aug. 1987.

[9] R. Venkataramanan, A. Sabanovic, and S. Cuk, "Sliding mode control of DC-to-DC converters," in Proc. IEEE IECON'85, 1985, pp. 251-258.

[10] E. Fossas, L. Martínez-Salamero, and J. Ordinas, "Sliding mode control reduces audio susceptibility and load perturbations in the Cuk converter," IEEE Trans. Circuits Syst., vol. 39, pp. 847-849, Oct. 1992.

[11] L. Martínez-Salamero, J. Calvente, R. Giral, A. Poveda, and E. Fossas, "Analysis of a bidirectional coupled-inductor cuk converter operating in sliding mode," IEEE Trans. Circuits Syst. I, vol. 45, pp. 355-363, Apr. 1998.

[12] K. Jezernik and D. Zadravec, "Sliding mode controller for a single phase inverter," in Proc. IEEE APEC'90, 1990, pp. 185-190.

[13] M. Carpita, M. Marchesioni, M. Oberti, and L. Puguisi, "Power conditioning system using sliding mode control," in Proc. IEEE PESC'88, 1988, pp. 623-633

[14] M. Carpita and M. Marchesoni, "Experimental study of a power conditioning using sliding mode control," IEEE Trans. Power Electron., vol. 11, pp. 731-742, Sept. 1996.

[15] F. Boudjema, M. Boscardin, P. Bidan, J. C. Marpinard, M. Valentin, and J. L. Abatut, "VSS approach to a full bridge Buck converter used for AC sine voltage generation," in Proc. IEEE IECON'89, 1989, pp. 82-89.

[16] E. Fossas and J. M. Olm, "Generation of signals in a buck converter with sliding mode control," in Proc. IEEE ISCAS'94, 1994, pp. 157-160.

[17] H. Pinheiro, A. S. Martins, and J. R. Pinheiro, "A sliding mode controller in single phase voltage source inverters," in Proc. IEEE IECON'94, 1994, pp. 394-398

[18] L. Malesani, L. Rossetto, G. Spiazzi, and A. Zuccato, "An AC power supply with sliding-mode control," IEEE Ind. Applicat. Mag., vol. 2, pp. 32-38, Jan./Feb. 1996.

[19] S. Jung and Y. Tzou, "Discrete sliding-mode control of a PWM inverter for sinusoidal output waveform synthesis with optimal sliding curve," IEEE Trans. Power Electron., vol. 11, pp. 567-577, July 1996.

[20] E. Fossas and D. Biel, "A sliding mode approach to robust generation on dc-to-dc converters," in Proc. IEEE CDC'96, 1996, pp. 4010-4012.

[21] E. Alarcón, D. Biel, F. Guinjoan, E. Fossas, E. Vidal, and A. Poveda, "Current-mode BiCMOS sliding-mode controller circuit for AC signal generation in switching power DC-DC converters," in Proc. IEEE MWSCAS'99, 1999, pp. 162-165.

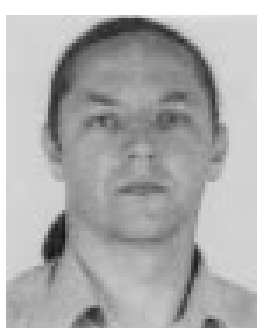

Enric Fossas received the Graduate and Ph.D. degrees in mathematics from the Universitat de Barcelona, Barcelona, Spain, in 1981 and 1986, respectively.

Since 1981, he has taught mathematics at the Universitat de Barcelona and mathematics and automatic control at the Universidad Politècnica de Catalunya, Barcelona, Spain, where he is currently an Assistant Professor. His research interests are nonlinear systems and mathematical control theory.

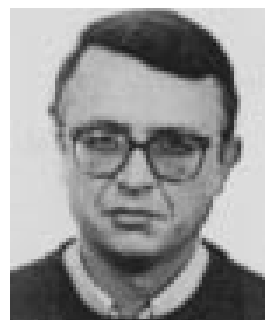

Francisco Guinjoan (M'92) received the Ingeniero de Telecomunicación and Doctor Ingeniero de Telecomunicación degrees from the Universitat Politècnica de Catalunya, Barcelona, Spain, and the Docteur es Sciences degree from the Université Paul Sabatier, Toulouse, France, in 1984, 1990, and 1992, respectively.

From 1984 to 1995 , he taught control theory and analog electronics at the Escuela Universitaria Politècnica de Vilanova i la Geltrú. He is currently an Associate Professor in the Departamento de Ingenieria Electrónica at the Escuela Técnica Superior de Ingenieros de Telecomunicación de Barcelona, Universitat Politècnica de Catalunya, Barcelona, Spain, where the teaches power electronics. His research interests include power electronics modeling, nonlinear circuit analysis, and analog circuit design.

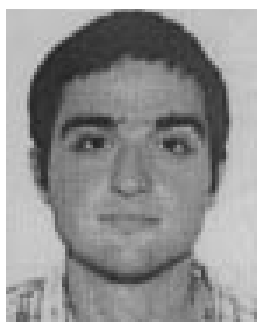

Eduard Alarcón (S'96-M'01) received the M.S (with national award) and Ph.D. degrees in electrical engineering from the Universitat Politecnica de Catalunya, Barcelona, Spain, in 1995 and 2000, respectively.

Since 1997, he has been an Assistant Professor in the Departamento de Ingenieria Electrónica, Escuela Técnica Superior de Ingenieros de Telecomunicación de Barcelona, Universitat Politècnica de Catalunya, Barcelona, Spain. His current research interests include analog and mixed-signal microelectronics, in particular, current-mode design, theoretical aspects of fuzzy control, switching $\mathrm{dc}-\mathrm{dc}$ converters, and nonlinear controller VLSI implementations. He has authored or coauthored more than 30 papers published in conference proceedings.

Prof. Alarcón was the recipient of the Myril B. Reed Best Paper Award at the IEEE Midwest Symposium on Circuits and Systems in 1998. He received a Fellowship from the Catalan Government from 1995 to 1997.

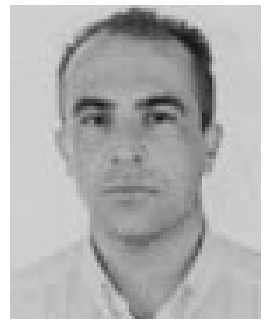

Alberto Poveda (M'98) received the Ingeniero de Telecomunicación and Doctor Ingeniero de Telecomunicación degrees from the Universitat Politècnica de Catalunya, Barcelona, Spain, in 1978 and 1988, respectively.

$\mathrm{He}$ is currently an Associate Professor in the Departamento de Ingenieria Electrónica, Escuela Técnica Superior de Ingenieros de Telecomunicación de Barcelona, Universitat Politècnica de Catalunya, Barcelona, Spain, where he teaches analog circuit design and power electronics. His research interests include analog circuit design and power processing modeling, control, and simulation. search interests include power electronics and non- 